\title{
Patience pays in spinal repair
}

\begin{abstract}
Steven A. Goldman
Center for Translational Neuromedicine, University of Rochester Medical Center, Rochester, New York, USA. Faculty of Health and Medical Sciences, University of Copenhagen and Rigshospitalet, Copenhagen, Denmark.
\end{abstract}

\begin{abstract}
Transplantation of human neural stem cells has long been proposed as a potential strategy for treating CNS injury and disease; however, application of this approach has had limited therapeutic benefit. Yet compared with rodents and other experimental mammals, humans have a relatively long time window for development of the brain and spinal cord. In this issue of the $J C l, L u$ and colleagues asked whether the results of neural stem cell transplantation might be improved by accommodating the protracted development of human neural cells. They used a rodent model of spinal cord injury, in which human neural progenitor cells were transplanted at the site of damage. While there was no observable benefit at early time points after transplantation, both anatomic and functional improvements in the injured animals emerged over the course of a year. In particular, the human progenitor cell population differentiated, matured, and integrated into the rodent spinal cords over a time frame that aligned with the normal development of these cells in humans. This study demonstrates that neural stem cells may offer significant therapeutic benefit after CNS injury; however, this process may take time and demands patience on the part of investigators, patients, and clinicians alike.
\end{abstract}

\section{Neuronal developmental time frames: humans versus other mammals}

Among mammals, humans have relatively long gestations, and within this period, the development of the brain is slow relative to that of other organs. Additionally, the maturation of the major macroglial cell types, astrocytes and oligodendrocytes, is especially prolonged. Human neurons and their synaptic networks mature over several years postnatally, while both glial recruitment and the functional maturation of the central white matter continue through childhood, well into adolescence and beyond $(1,2)$. Yet, despite these clear and obvious differences in human biology relative to experimental rodents, little weight has traditionally been given to the importance of these vastly different developmental time frames in modeling cell therapeutics.

In this issue, a group led by Mark Tuszynski (3) apply the knowledge of basic human development to the use of transplanted human neural stem cells in the treatment of spinal cord injury (SCI). Specifically, Lu et al. (3) approached the long-vexing problem that there is limited recovery associated with cell therapeutics used for SCI by investigating whether a longer wait is needed to see therapeutic benefit when using human neural stem cells. In essence, the issue boils down to whether human cells transplanted into a rodent model behave as other cells in their host, in terms of their maturation rate, or whether they do so as if they were in a

Related Article: p. 3287

Conflict of interest: S.A. Goldman is a consultant to Healios KK (Japan), receives corporate funding from NovoSeeds (Denmark), and holds multiple patents in regard to neural and glial progenitor cell-based therapeutics for disorders of the CNS, including US patents 9,709,553; 9,371,513; 8,206,699; 8,263,402; 9,724,432; and 7,524,491.

Reference information: / Clin Invest. 2017;127(9):3284-3286. https://doi.org/10.1172/JCI96650. human context, by maturing at a slower rate. This question is deceptively simple but has profound therapeutic implications. If the development of transplanted human neural cells spans time frames associated with normal human development, then far longer observation times than have traditionally been used in experimental rodent models would be required to adjudge transplant success or failure, whether in the lab or in the clinic.

$\mathrm{Lu}$ et al. transplanted neural stem cells derived from human embryonic stem cells into mice with established lesions in the cervical spinal cord, two weeks after cord hemisection. In this model of SCI, ipsilateral improvement is typically minimal, such that any improvement may be reasonably associated with therapeutic intervention. $\mathrm{Lu}$ and colleagues carefully assessed the proliferation, maturation rates, expansion dynamics, and differentiated fates of the human-derived cells and their progeny. The human neural stem and progenitor cells, which were not operationally distinguished, slowly but surely gave rise to neurons, astrocytes, and oligodendrocytes that matured and did so coincidentally with a fall in the mitotic index of their parental progenitor cells. The maturation of neurons took months, while astrocytic development took at least half a year, and oligodendrocytes continued to develop and produce myelin for well over a year after transplantation (Figure 1). These observations all coincide with time courses that have been described by others using human neural progenitors in vitro, and they roughly correspond to the developmental ontogeny of each cell type in vivo during normal human development.

\section{Improvement takes time}

Remarkably, as the human donor cells dispersed, matured, and made connections, both with one another and with host neurons, the injured animals began to exhibit functional improvement; however, when viewed from the perspective of normal human development, this time frame to 
NSC

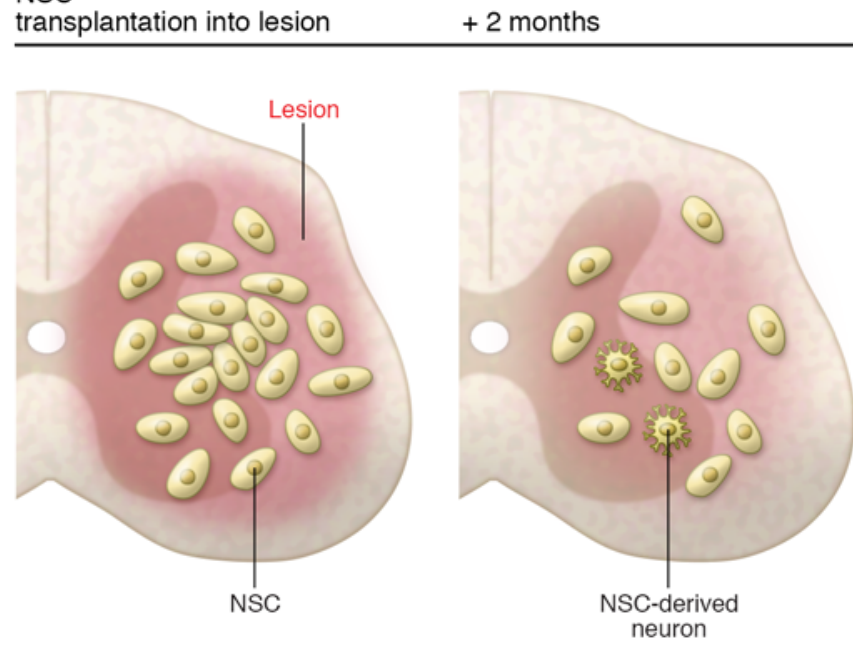

Figure 1. The gradual differentiation of human neural stem cells into neurons and glia following transplantation into the injured spinal cord. Human neural stem cells (NSCs) grafted into spinal cord lesions follow a developmental time course that is similar to that observed during human development. Stem cell-derived neurons first appear at the site of transplantation by 2 months after transplantation, while astrocytes appear by 6 months. By that point, connections between host neurons and donor-derived neurons are first seen as well. By a year after transplantation, NSC-derived myelinogenic oligodendrocytes are observed, while functional improvement is evident in the transplanted animals relative to their nonengrafted controls.

improvement was largely predictable. The functional increments seen in treated animals relative to rodents that received fibrin gel-only vehicle-implanted controls were not observed until over a year after introduction of the human neural stem cells. By that time, the donor-derived human neurons had differentiated into the major neuronal spinal phenotypes and had produced astrocytes as well as oligodendrocytes. In particular, the donor-derived neurons dispersed, following the initial migration routes of their progenitors, beyond a centimeter from their injection sites. Additionally, the number of human-derived neurons recovered, following an initial decrease. Both astrocytes and oligodendrocytes were also produced, although neither the functionality of the former nor the myelination competence of the latter was assessed.

With these observations, Lu et al. make a compelling case that human neural stem cell grafts can indeed mediate improvement in both anatomic circuit reconstruction and functional status after SCI; however, graft-associated increments in recovery may have extremely prolonged time courses. The time to notable improvement observed by $\mathrm{Lu}$ and colleagues is certainly congruent with our understanding of the time course of human neuronal and glial development. Surprisingly, though, these species-specific time frames have never before been systematically considered when interpreting the results of human neural grafts into rodent models of SCI, or into any other models of CNS injury.

To be sure, the slow maturation of human neural cells after experimental xenografting has been noted in a variety of other settings by a number of authors. Brustle, Zhang, and colleagues, in some of the first studies of human cell chimerization into the brains of rodents, reported the relatively slow time course of human neuronal maturation in the otherwise rapidly developing rodent host environment (4, 5). Windrem and colleagues subsequently revealed that human macroglia have a far more delayed maturation, exceeding six to nine months, compared with that of neurons, in a study that described the time courses of human astroglial and oligodendroglial maturation after xenograft into the rodent brain $(6,7)$. Windrem in particular noted that after xenografting human glial progenitor cells into congenitally hypomyelinated shiverer mice, oligodendrocytic differentiation and forebrain myelination took as long as a year. Similarly, Han and colleagues noted that human astrocytic progenitor engraftment could substantially influence the cognitive capabilities of normal mice; however, this effect did not emerge for seven to nine months after neonatal graft, reflecting the slow maturation of the implanted human glia (8). Analogous studies of human neuronal progenitors into the primate brain (9) have similarly revealed the extended nature of human neuronal and glial maturation after transplantation, reflecting the largely cell-autonomous nature of neural differentiation.

Despite the extensive literature examining the slow, cell-autonomous maturation of human neural phenotypes after transplantation, this knowledge had not previously been translated to studies of neuronal replacement in the structural repair of the injured brain or spinal cord. As such, the study by Lu et al. is a fundamentally important contribution, as it alerts the broader field of experimental modeling of CNS disease to a significant consideration in the use of human neural cells as therapeutic vectors. Specifically, this work confirms that human neural progenitors take a long time to mature and integrate, over a period that is far longer than that observed in their rodent homologs, and that this extended time course needs to be considered in both the design and interpretation of future preclinical and clinical studies alike.

\section{The steps ahead}

Future studies will no doubt address the phenotypic heterogeneity, physiological competence, and functional network 
integration of late-generated human neurons and glia. A variety of techniques, both neurophysiologic and anatomic, including rabies tracing of anatomic connections as well as opto- and chemogenetic direction of neuronal activity, are now available and should permit intense interrogation of the new spinal networks formed by these transplants. Single-cell transcriptomics of the donor cells within the transplanted spinal cords should similarly permit determination of the heterogeneity of neuronal and glial phenotypes generated from human neural progenitors and whether that range reflects the diversity of phenotypes of the normal mature spinal cord.

In addition to the need to establish the functional neuroanatomy of donor-derived neuronal and glial integration over time, future studies will also need to rigorously define the relative clinical advantage of transplanting cells after SCI and then waiting, versus just waiting. Patients with SCI, at least those with nontransective and incomplete segmental spinal loss, can improve over time and may do so spontaneously; their improvement may be as significant as it is unpredictable. Indeed, the slow improvement of these individuals mimics that of patients with traumatic brain injury and stroke, analogous conditions for which the extent of clinical recovery over long time periods can often be shocking. The nature of the slow, spontaneous improvement in these conditions remains enigmatic, though it is no doubt a combination of network reorganization, functional compensation, glial replacement from endogenous progenitors (10), and, conceivably, neuronal replacement (11). As such, Lu et al. and others in the field will need to establish the superiority of cell transplantation over more conser- vative management strategies. Moreover, the durability of that relative benefit over long periods of time will need to be established. Doing so will in turn require the development of rigorous inclusion and exclusion criteria for defining appropriate transplant recipients, as well as predictive metrics for assessing the likely prognosis soon after injury. Yet, despite these challenges, the exciting data presented by $\mathrm{Lu}$ and colleagues, contrasted with the limited recovery and dearth of alternatives for most SCI patients, bode exceedingly well for the value of this treatment strategy going forward. Future studies will no doubt focus on these issues as logical next steps for this provocative work as it advances to the clinic.

Lu et al. have thus done a great service to investigators in this field, as well as to their future patients, with this provocative report. By highlighting the slow but steady nature of human donor cell maturation and circuit integration, and by convincingly demonstrating that transplant-based circuit reconstruction in the injured spinal cord is feasible and effective, this study has advanced the cause of cell replacement therapy, for spinal repair as well as for other structural disorders of the CNS In doing so, this work has reiterated that in cell therapeutics, as in so many other domains of medicine, all good things come to those who wait.

\section{Acknowledgments}

S.A. Goldman is supported by National Institute of Neurological Disorders and Stroke (NINDS) grants R01NS75345 and R01NS100366, and National Institute of Mental Health (NIMH) grants R01MH104701 and R01MH099578; the Adelson Medical Research Foundation; the CHDI Foundation; the Novo Nordisk Foundation; the Lundbeck Foundation; the
ALS Association; NovoSeeds; and the Progressive Multifocal Leukoencephalopathy (PML) Consortium.

Address correspondence to: Steven A. Goldman, University of Rochester Medical Center, 601 Elmwood Ave, Rochester, New York 14642, USA. Phone: 585.275.0550; Email: steven_goldman@urmc.rochester. edu orgoldman@sund.ku.dk.

1. McKenzie IA, et al. Motor skill learning requires active central myelination. Science. 2014;346(6207):318-322.

2. Sigaard RK, Kjær M, Pakkenberg B. Development of the cell population in the brain white matter of young children. Cereb Cortex. 2016;26(1):89-95.

3. Lu P, et al. Prolonged human neural stem cell maturation supports recovery in injured rodent CNS. JClin Invest. 2017;127(9):3287-3299.

4. Brüstle $\mathrm{O}$, et al. Chimeric brains generated by intraventricular transplantation of fetal human brain cells into embryonic rats. Nat Biotechnol. 1998;16(11):1040-1044.

5. Zhang SC, Wernig M, Duncan ID, Brüstle $\mathrm{O}$, Thomson JA. In vitro differentiation of transplantable neural precursors from human embryonic stem cells. Nat Biotechnol. 2001;19(12):1129-1133.

6. Windrem MS, et al. Fetal and adult human oligodendrocyte progenitor cell isolates myelinate the congenitally dysmyelinated brain. Nat Med. 2004;10(1):93-97.

7. Windrem MS, et al. Neonatal chimerization with human glial progenitor cells can both remyelinate and rescue the otherwise lethally hypomyelinated shiverer mouse. Cell Stem Cell. 2008;2(6):553-565.

8. Han X, et al. Forebrain engraftment by human glial progenitor cells enhances synaptic plasticity and learning in adult mice. Cell Stem Cell. 2013;12(3):342-353.

9. Ourednik V, et al. Segregation of human neural stem cells in the developing primate forebrain. Science. 2001;293(5536):1820-1824.

10. Franklin RJ, Ffrench-Constant C. Remyelination in the CNS: from biology to therapy. Nat Rev Neurosci. 2008;9(11):839-855.

11. Magavi SS, Leavitt BR, Macklis JD. Induction of neurogenesis in the neocortex of adult mice. Nature. 2000;405(6789):951-955. 